\title{
Detection of Listeria monocytogenes in Humans, Animals and Foods
}

\author{
Takashi IIDA, Masako KANZAKI, Akiko NAKAMA, Yataro KOKUBO, Tsutomu MARUYAMA ${ }^{1)}$ and \\ Choji KANEUCHI ${ }^{2}$ \\ The Tokyo Metropolitan Research Laboratory of Public Health, 3-24-1 Hyakunin-cho, Shinjuku-ku, Tokyo 169-0073 and ${ }^{11}$ School of \\ Environmental Health, Department of Food Hygiene and ${ }^{2}$ School of Veterinary Medicine, Second Department of Veterinary Public \\ Health, Azabu University, Sagamihara, Kanagawa 229-8501, Japan
}

(Received 7 November 1997/Accepted 14 July 1998)

ABSTRACT. The Listeria monocytogenes-carrying rates were $100 \%$ for listeriosis patients and $1.3 \%$ for healthy humans. The $L$. monocytogenes contamination rates for retail sliced beef $(34.2 \%)$ and pork $(36.4 \%)$ were significantly higher $(\mathrm{p}<0.05)$ than those for cattle $(2.0 \%)$ and pigs $(0.8 \%)$ and for cattle $(4.9 \%)$ and swine $(7.4 \%)$ carcasses. The percentages of serotypes $1 / 2 \mathrm{a}, 1 / 2 \mathrm{~b}$ and $4 \mathrm{~b}$ which are most dominant in human patients were high in isolates from fresh $(90.0 \%)$ and processed (100\%) fish and shellfish and imported natural cheese (96.7\%). — KEY wORDS: Listeria monocytogenes, meat, serotype

J. Vet. Med.Sci.60(12): 1341-1343, 1998

A number of cases of listeriosis caused by the intake of foods such as cheese, meat, ice cream and fish have been reported overseas since the beginning of 1980s [6, 8, 10] and Listeria monocytogenes serotypes $1 / 2 \mathrm{a}, 1 / 2 \mathrm{~b}$ and $4 \mathrm{~b}$ have been almost exclusively implicated in these incidents. Thus, listeriosis has been positioned as one of the important foodborne infections [14]. Although some 30 sporadic cases of listeriosis have been confirmed every year [3], their sources of infection are mostly unknown and such foodborne cases have not yet been reported in Japan. Therefore, it is necessary to investigate in detail the scope and degree of food contamination with $L$. monocytogenes. In this study, detection of L. monocytogenes was attempted on a large number of samples collected widely from humans including listeriosis patients, animals and foods in Japan and the isolates were serotyped on the basis of cellular and flagellar antigens.

For a period from 1988 through 1994, a total of 31,127 samples from listeriosis patients, healthy humans, animals, meat, fish and cheese were subjected to isolation of L. monocytogenes (Table 1). Blood and spinal fluid specimens were collected from listeriosis patients who were mainly the aged and hospitalized in Tokyo and its vicinity. Stool specimens were collected from healthy humans consisting of school children, junior and senior high school students and office workers in Tokyo and dogs at the animal protection centers in Tokyo. Intestinal contents were collected from rats (Rattus rattus) captured at restaurants in buildings in downtown Tokyo. The contents of large intestine healthy cattle and pigs brought into slaughterhouses throughout Japan were sampled. Surface swabs were collected from cattle and swine carcasses at wholesale markets in several regions of Japan. The surface of an area of $100 \mathrm{~cm}^{2}$ mainly the neck of carcasses was swabbed with sterilized gauze. Sliced beef, pork, chicken and horse meat were collected at supermarkets in Tokyo and other prefectures. Fresh and processed fish and shellfish specimens were collected at the municipal fish markets in Tokyo. Samples of imported natural cheese were obtained at department stores in Tokyo.
A 1-g portion of stool and intestinal content samples was enriched in $9 \mathrm{~m} l$ UVM medium (Difco Laboratories, Detroit, Mich.) or EB medium (Difco). The swabs were each added to $9 \mathrm{~m} l$ of an enrichment medium. Samples of natural cheese (25 g) and meat and fresh and processed seafoods (10 $\mathrm{g}$ each) were each placed in a stomacher bag containing $225 \mathrm{~m} l$ or $90 \mathrm{~m} l$ of the enrichment medium, and homogenized for $1 \mathrm{~min}$ with a stomacher. The stomacher bags were incubated at $30^{\circ} \mathrm{C}$ for $48 \mathrm{hr}$. Two to three loopfuls of the cultured enrichment medium from the stomacher bags were streaked on a plate of Oxford agar medium (Oxoid, Unipath Ltd., Basingstoke, Hampshire, England) or PALCAM agar medium (Merck Co., Inc., Rahway, N.J.) supplemented with amphotericin B (Sigma Chemical Co., St. Louis, Mo.) at a concentration of $6 \mu \mathrm{g} / \mathrm{m} l$ to inhibit the growth of mycetes and the plates were incubated at $30^{\circ} \mathrm{C}$ for $48 \mathrm{hr}$. Slightly flat colonies with brownish color in the periphery, which is characteristic of Listeria, were subcultured on Tryptose Agar medium (Difco). The colonies developed on Tryptose Agar medium were observed under a low power microscope with obliquely reflected light and blue greenish colonies were selected [12]. Identification was carried out by Gram staining, catalase and VP tests, observation of umbrella-like growth and motility in semiliquid Brain Heart Infusion Agar medium (Difco), utilization of rhamnose, xylose and mannitol, $\beta$ hemolysis on 5\% sheep blood Tryptic Soy Agar medium (Difco) and the CAMP test [12] with Staphylococcus aureus JTE 88-221 and Rhodococcus equi JTE 89-387 .

The factor sera were prepared by hyperimmunizing rabbits (Japan Laboratory Animals, Inc., Tokyo) with each of 12 reference strains, L. monocytogenes JTE 88-178 to JTE $88-180$ and JTE $88-188$ to JTE $88-191$, by the method of Seeliger and Höhne [11]: 11 kinds of somatic $(\mathrm{O})$ antigen factor sera and four kinds of flagellum $(\mathrm{H})$ antigen factor sera were obtained. Serotyping was conducted by $\mathrm{O}$ and $\mathrm{H}$ agglutination reactions on microplates [1]. Description of the serotypes of the isolates was followed after Seeliger and Höhne [11].

As shown in Table 1, the L. monocytogenes-carrying rates 
Table 1. Detection of L. monocytogenes in humans, animals and foods

\begin{tabular}{|c|c|c|c|c|c|c|c|c|}
\hline & \multirow{2}{*}{ Samples } & \multirow{2}{*}{$\begin{array}{c}\text { No. of } \\
\text { samples } \\
\text { examined }\end{array}$} & \multirow{2}{*}{$\begin{array}{l}\text { No. of } \\
\text { samples } \\
\text { ositive }(\%)\end{array}$} & \multicolumn{5}{|c|}{ No. of isolates ${ }^{a)}(\%)$} \\
\hline & & & & $1 / 2 \mathrm{a}$ & $1 / 2 b$ & $1 / 2 \mathrm{c}$ & $4 \mathrm{~b}$ & Others \\
\hline Listeriosis patient & Blood or spinal fluid & 39 & $39(100)$ & $10(25.6)$ & $7(18.0)$ & 0 & $22(56.4)$ & 0 \\
\hline Healthy human & Feces & 2,970 & $38(1.3)$ & $17(44.7)$ & $9(23.7)$ & 0 & $10(26.3)$ & $2(5.3)$ \\
\hline \multicolumn{9}{|l|}{ Animal and meat } \\
\hline Rat & Content of large intestine & 199 & $13(6.5)$ & $2(15.4)$ & $4(30.8)$ & $1(7.6)$ & $2(15.4)$ & $4(30.8)$ \\
\hline Dog & Feces & 540 & $5(0.9)$ & 0 & 0 & $1(20.0)$ & $2(40.0)$ & $2(40.0)$ \\
\hline Cattle & Content of large intestine & 9,539 & $189(2.0)$ & $4(2.1)$ & $12(6.3)$ & $150(78.5)$ & $3(1.6)$ & $20(10.5)$ \\
\hline Cattle carcass & Swab of surface & 4,106 & $202(4.9)$ & $3(1.5)$ & $11(5.4)$ & $179(88.6)$ & $3(1.5)$ & $6(3.0)$ \\
\hline Beef & Sliced & 225 & $77(34.2)^{\mathrm{b})}$ & $11(14.3)^{b)}$ & $12(15.6)$ & $33(42.8)$ & $18(23.4)^{\mathrm{b})}$ & 3 (3.9) \\
\hline Pig & Content of large intestine & 5,975 & $46(0.8)$ & $11(23.9)$ & $15(32.6)$ & $5(10.8)$ & $1(2.2)$ & $14(30.5)$ \\
\hline Swine carcass & Swab of surface & 4,330 & $321(7.4)$ & $18(5.6)$ & $26(8.1)$ & $265(82.6)$ & $9(2.8)$ & $3(0.9)$ \\
\hline Pork & Sliced & 209 & $76(36.4)^{c)}$ & $11(14.5)$ & $16(21.1)$ & $42(55.2)$ & $6(7.9)$ & $1(1.3)$ \\
\hline Chicken & Sliced & 328 & $123(37.5)$ & $49(39.8)$ & $27(22.0)$ & $31(25.2)$ & $15(12.2)$ & $1(0.8)$ \\
\hline Horse meta & Sliced & 497 & $15(3.0)$ & $5(33.3)$ & $5(33.3)$ & $4(26.7)$ & 0 & $1(6.7)$ \\
\hline \multicolumn{9}{|l|}{ Fish and shellfish } \\
\hline Fresh & Fish, shellfish, shurimp, etc & 781 & $10(1.3)$ & $2(20.0)$ & $2(20.0)$ & 0 & $5(50.0)$ & $1(10.0$ \\
\hline Processed & Boiled shellfish, Smoked salmon, etc & 247 & $13(5.3)$ & $7(53.8)$ & $5(38.5)$ & 0 & $1(7.7)$ & 0 \\
\hline Cheese & Imported natural cheese & 1,142 & $30(2.6)$ & $10(33.3)$ & $11(36.7)$ & $1(3.3)$ & $8(26.7)$ & 0 \\
\hline
\end{tabular}

a) One strain was isolated from one positive sample. b) Significantly different from cattle and cattle carcasses at $\mathrm{P}<0.05$ by $\mathrm{X}^{2}$ test. c) Significantly different from pigs and swine carcasses at $\mathrm{P}<0.05$ by $\mathrm{X}^{2}$ test.

were $100 \%$ and $1.3 \%$ for listeriosis patients and stool specimens of healthy humans, respectively. In animals examined, the rates were $0.9,2.0$ and $0.8 \%$ for dogs, cattle and pigs, respectively. Rats showed a relatively high rate being $6.5 \%$. In foods, the contamination rates were $3.0,7.4$ and $3.0 \%$ for cattle and swine carcasses and sliced horse meat, respectively, and $2.6 \%$ for cheese and 1.3 and $5.3 \%$ for fresh and processed fish and shellfish, respectively. Sliced beef, pork and chicken showed considerably high rates of $34.2,36.4$ and $37.5 \%$, respectively.

A total of 1,195 isolates were classified into 15 serotypes and four serotypes $1 / 2 \mathrm{a}, 1 / 2 \mathrm{~b}, 1 / 2 \mathrm{c}$ and $4 \mathrm{~b}$ were most prevalent accounting for $95.1 \%$ of the total isolates. Isolates from listeriosis patients were classified exclusively into three serotypes $1 / 2 \mathrm{a}, 1 / 2 \mathrm{~b}$ and $4 \mathrm{~b}$. The isolates from stool specimens of healthy humans, cheese, fish and shellfish were almost exclusively identified as one of the three serotypes and those from animals and raw meats were mostly identified into the four prevalent serotypes. The serotypes other than the four prevalent ones were 1, 3b, 3c, 4a, 4ab, $4 \mathrm{~b}(\mathrm{x}), 4 \mathrm{c}, 4 \mathrm{~d}, 4 \mathrm{e}, 4 \mathrm{~g}$ and 4 . Meat and fish were contaminated with $L$. monocytogenes three serotypes $1 / 2 \mathrm{a}$, $1 / 2 \mathrm{~b}$ and $4 \mathrm{~b}$ with the progress of their processing steps.

In this study, a large number of samples collected widely from various sources in Japan were investigated for L. monocytogenes. Processed raw meat, fish and shellfish samples were included in this study to examine for the influence of processing on the contamination with L. monocytogenes. The L. monocytogenes contamination rates of the stool specimens of healthy humans, intestinal contents of animals, livestock foodstuffs and seafoods were essentially similar to those reported in other countries [7].

The primary source of $L$. monocytogenes contamination of carcasses is assumed to be the contents of the large intestine of slaughtered animals [13]. In this study, although it is not proper to compare the L. monocytogenes contamination rates of carcasses (surface swabs) and feces and retail sliced meat (in grams), as their sampling methods were different from each other, the contamination rates of the retail sliced beef $(34.2 \%)$ and pork $(36.4 \%)$ were significantly higher $(\mathrm{p}<0.05)$ than those of the cattle $(4.9 \%)$ and swine $(7.4 \%)$ carcasses and of the intestinal contents of cattle $(2.0 \%)$ and pigs $(0.8 \%)$. Similarly, the percentage of each of the three serotypes $1 / 2 \mathrm{a}, 1 / 2 \mathrm{~b}$ and $4 \mathrm{~b}$, which are main causal serotypes of listeriosis, was higher $(p<0.05)$ in isolates from sliced beef than in those from intestinal contents of cattle and cattle and swine carcasses.

In pig intestinal isolates, the percentages of the three serotypes were considerably high; however, the rates were lowered in isolates from swine carcasses to the level similar to those in isolates from cattle carcasses and then became high in sliced pork isolates to the level similar to those in sliced beef isolates. These results indicate that raw meat is contaminated with L. monocytogenes three serotypes $1 / 2 \mathrm{a}$, $1 / 2 \mathrm{~b}$ and $4 \mathrm{~b}$ with the progress of processing steps and that, like the poultry-processing environment concerning $L$. monocytogenes contamination [5], slaughterhouses and meat processing environments are more important as a source of 
L. monocytogenes contamination of the meat than intestinal contents of slaughtered animals.

Caution is needed in handling retail meat products as they can be a potent source of contamination leading to listeriosis. However, retail raw meat products are less likely to be the direct source of infection so long as their handling and method of cooking are adequate and since meat products are usually ingested after cooking and the bacterial content per gram is often less than $10^{2}[4,9]$.

L. monocytogenes contamination rate of retail sliced horse meat was extremely low as compared with the corresponding retail sliced beef, pork or chicken. This may suggest that much attention is being paid to the processing of horse meat to avoid bacterial contamination because it is often eaten raw as a delicacy in Japan.

The L. monocytogenes-carrying rate $(6.5 \%)$ in rats was higher $(\mathrm{p}<0.05)$ than those of $\operatorname{dogs}(0.9 \%)$, cattle $(2.0 \%)$ or pigs $(0.8 \%)$. Furthermore, the percentage $(61.5 \%)$ of the three serotypes $1 / 2 \mathrm{a}, 1 / 2 \mathrm{~b}$ and $4 \mathrm{~b}$, which often most cause human disease, in rat isolates was the highest among the animals examined other than healthy humans and the carrier rate $(4.0 \%)$ of the three serotypes in rats was also higher $(\mathrm{p}<0.05)$ than those in such other animals examined as dogs $(0.4 \%)$, cattle $(0.3 \%)$ and pigs $(0.5 \%)$. Although approximately one thirds of human listeriosis cases in foreign countries are known to be foodborne, the remaining major cases are still unknown of the source of infection [9]. It might be possible to consider that rats play a role as a contaminator in the environment such as restaurants in the buildings in cities.

The L. monocytogenes contamination rate (1.3\%) of fresh fish and shellfish was rather low in comparison with that (about 36\%) for the retail sliced meat. The bacterial count per gram of fresh fish and shellfish has been reported to be as low as less than $10^{2}$ [9], in contrast to $10^{3}$ to $10^{7}$ in cheese [9] which has often been regarded to be closely associated with the incidence of listeriosis. Moreover, fresh seafoods eaten without cooking is rarely stored for an extended period of time and the bacterial content in such seafoods at the time of ingestion could seldom be high even if L. monocytogenes is capable of proliferation at low temperatures. Therefore, fresh seafoods will seldom be the source of infection with $L$. monocytogenes.

As for processed seafoods that are ready-to-serve without cooking such as smoked or flaked salmon, boiled shellfish and so forth, the L. monocytogenes contamination rate $(5.3 \%)$ was low as compared with those of retail sliced meat. However, the isolates from processed seafoods all belonged to serotypes $1 / 2 \mathrm{a}, 1 / 2 \mathrm{~b}$ or $4 \mathrm{~b}$. Furthermore, processed seafoods are kept refrigerated for a relatively long period of time like natural cheese and L. monocytogenes may grow during the storage. Therefore, the processed seafoods may be a possible source of L. monocytogenes infection. This has already been pointed out also by Jemmi [2].

The three serotypes of $L$. monocytogenes $1 / 2 \mathrm{a}, 1 / 2 \mathrm{~b}$ and $4 \mathrm{~b}$ are considered to be of particular importance as causal serotypes of listeriosis since their percentages are overwhelmingly high in the isolates clinically collected from diseased animals and human patients [8]. In this study, Japanese isolates derived from patients were also all classified into any one of these serotypes. Although the L. monocytogenes-carring rates of stool specimens of healthy humans was similar to those of other animals examined, such a high rate as $94.7 \%$ of healthy human isolates were classified into the three serotypes, suggesting that if the high risk groups such as infants, aged people and those with immunodeficiency become carriers of L. monocytogenes, chances are that they are likely to develop listeriosis.

\section{REFERENCES}

1. Iida, T., Kanzaki, M. and Maruyama T. 1995. Ann. Rep. Tokyo Metr. Res. Lab. Public Health 46: 46-50 (in Japanese).

2. Jemmi, T. 1993. Arch. Lebensm. Hyg. 44: 10-13.

3. Kokubo, Y. 1992. Jpn. J. Food Microbiol. 9: 13-22 (in Japanese with English summary).

4. Kokubo, Y., Kaneko, S., Iida, T. and Maruyama, T. 1992. Ann. Rep. Tokyo Metr. Res. Lab. Public Health 43: 82-86 (in Japanese with English summary).

5. Lawrence, L. M. and Gilmour, A. 1995. Appl. Environ. Microbiol. 61: 2139-2144.

6. Linnan, M. J., Mascola, L., Lou, X. D., Goulet, V., May, S., Salminen, C., Hird, D. W., Yonekura, M. L., Hayes, P., Weaver, R., Audu- rier, A., Plikaytis, B. D., Fannin, S. L., Kleks, A. and Broome, C.V. 1988. New Engl. J. Med. 319: 823-828.

7. Low, J. C. and Donachie, W. 1997. Vet. J. 153: 9-20.

8. McLauchlin, J. 1997. Rev. Med. Microbiol. 8: 1-14.

9. Rocourt, J. and Cossart, P. 1997. pp. 337-352. In: Food Microbiology, Fundamentals and Frontiers (Doyle, M. P., Beuchat L. R. and Montville T. J. eds.), AMS Press, Washington, D.C.

10. Schlech, W. F., Lavigne, P. M., Boetolussi, R. A., Allen, A. C., Haldane, E. V., Wort, A. J., Hightower, A. W., Johnson, S. E., King, S. H., Nicholls, E. S. and Broome, C. V. 1983. New Engl. J. Med. 308: 203-206.

11. Seeliger, H. P. R. and Höhne, K. 1979. pp. 31-49. In: Methods in Microbiology (Bergan, T. and Norris, J. R. eds.), Academic Press, New York.

12. Seeliger, H. P. R. and Jones, D. 1986. pp. 1235-1245. In: Bergey's Manual of Systematic Bacteriology, 9th ed. (Sneath, P. H. A., Mair, N. S., Sharpe, M. E. and Holt, J. G. eds.), William and Wilkins, Baltimore.

13. Skovgaard, N. and Norrung, B. 1989. Int. J. Food Microbiol. 8: 59-63.

14. WHO Working Group. 1988. Bull. WHO. 66: 421-428. 